\title{
Network pharmacology-based strategy to investigate pharmacological mechanisms of Zuojinwan for treatment of gastritis
}

\author{
Guohua Yu', Wubin Wang ${ }^{1}$, Xu Wang ${ }^{1}$, Meng Xu' ${ }^{1}$ Lili Zhang ${ }^{1}$, Lei Ding ${ }^{1}$, Rui Guo ${ }^{1}$ and Yuanyuan Shi ${ }^{1,2^{*}}$
}

\begin{abstract}
Background: Zuojinwan (ZJW), a classic herbal formula, has been extensively used to treat gastric symptoms in clinical practice in China for centuries. However, the pharmacological mechanisms of ZJW still remain vague to date.

Methods: In the present work, a network pharmacology-based strategy was proposed to elucidate its underlying multi-component, multi-target, and multi-pathway mode of action against gastritis. First we collected putative targets of ZJW based on TCMSP and STITCH databases, and a network containing the interactions between the putative targets of ZJW and known therapeutic targets of gastritis was built. Then four topological parameters, "degree", "betweenness", "closeness", and "coreness" were calculated to identify the major targets in the network. Furthermore, the major hubs were imported to the Metacore database to perform a pathway enrichment analysis.

Results: A total of 118 nodes including 59 putative targets of ZJW were picked out as major hubs in terms of their topological importance. The results of pathway enrichment analysis indicated that putative targets of ZJW mostly participated in various pathways associated with anti-inflammation response, growth and development promotion and G-protein-coupled receptor signaling. More importantly, five putative targets of ZJW (EGFR, IL-6, IL-1 $\beta$, TNF- $\alpha$ and MCP-1) and two known therapeutic targets of gastritis (CCKBR and IL-12ß) and a link target NF$\mathrm{KB}$ were recognized as active factors involved in the main biological functions of treatment, implying the underlying mechanisms of ZJW acting on gastritis.
\end{abstract}

Conclusion: ZJW could alleviate gastritis through the molecular mechanisms predicted by network pharmacology, and this research demonstrates that the network pharmacology approach can be an effective tool to reveal the mechanisms of traditional Chinese medicine (TCM) from a holistic perspective.

Keywords: Zuojinwan, Network pharmacology, Gastritis

\section{Background}

Gastritis is an acute or chronic, diffuse or focal inflation of the lining of the stomach [1]. It is brought on by many factors, including infection by Helicobacter pylori, drug induced such as aspirin, Non-Steroidal Anti-Inflammatory Drugs (NSAIDs), corticosteroids and alcohol consumption [2]. The most frequent symptoms of gastritis include upper abdominal pain, heartburn, nausea, and vomiting [3, 4]. Gastritis is believed to affect about half of people in the world and it generates

\footnotetext{
* Correspondence: yshi@bucm.edu.cn

${ }^{1}$ School of Life Sciences, Beijing University of Chinese Medicine, No.11 East road, North 3rd Ring Road, Beijing 100029, China

${ }^{2}$ Shenzhen Hospital, Beijing University of Chinese Medicine, No. 1 Dayun road, Sports New City Road, Shenzhen 518172, China
}

considerable costs to society [5]. Although current therapies, including antacids, $\mathrm{H}-2$ blockers, proton pump inhibitors and antibiotics can alleviate some major symptoms of gastritis, these medications have also triggered a series of serious side effects like abdominal pain (or stomach pain), constipation, and diarrhea. Therefore, it is still necessary to find novel and safe prevention strategies.

Traditional Chinese medicine (TCM) is a comprehensive medicinal system that plays an important role in health maintenance for Asian people, and has gradually gained popularity in western countries due to the reliable therapeutic efficacy and fewer side effects $[6,7]$. Based on the theory of traditional Chinese herbal

(c) The Author(s). 2018 Open Access This article is distributed under the terms of the Creative Commons Attribution 4.0 International License (http://creativecommons.org/licenses/by/4.0/), which permits unrestricted use, distribution, and reproduction in any medium, provided you give appropriate credit to the original author(s) and the source, provide a link to the Creative Commons license, and indicate if changes were made. The Creative Commons Public Domain Dedication waiver (http://creativecommons.org/publicdomain/zero/1.0/) applies to the data made available in this article, unless otherwise stated. 
medical science, TCM offers bright prospects for the prevention and treatment of complex diseases such as gastritis in a systematic way [8].

Zuojinwan, which consists of $R$. coptidis and E. rutaecarpa powder in the ratio of $6: 1(w / w)$, is a famous Chinese medicine prescription used for treatment of gastric diseases $[9,10]$. ZJW was first recorded in a famous ancient medicine treatise Danxi Xinfa in Yuan Dynasty of Chinese history (1271 AD-1368 AD), and has been approved by the China Food and Drug Administration (CFDA). From the bench to the bedside, previous researches on ZJW for gastric diseases could be mainly documented into two sections: (1) Clinical practices show that ZJW has been widely used for treating gastric diseases. (2) Basic researches indicate that ZJW exerts a range of pharmacological activities, including anti-inflammation, anti-ulcer and anti-acid activities and inhibitory effect on the growth of Heliobacter pylori. Although well-practiced in clinical medicine, very little is known about the active substances and specific molecular mechanisms of ZJW acting on gastritis.

Similar to other TCM formulas, ZJW is a multi-component and multi-target agent that achieves its specific therapeutic efficacy through active components that regulate molecular networks within the body. Therefore, it is hard to investigate the pharmacological mechanisms of ZJW in the treatment of gastritis. With the rapid progress of bioinformatics, systems biology and polypharmacology, network pharmacology-based approaches have been proven to be a powerful way for compatible and mechanistic exploration of TCM formula $[7,11,12]$. For example, Yu et al. used a network pharmacology method to analyze the synergistic mechanism of Yin-Huang-Qing-Fei capsule acting on chronic bronchitis [13]. Yue et al. developed an integrated system pharmacology approach, combined a number of network-based computational methods and algorithmbased approaches to clarify the mechanisms of Danggui-Honghua for treatment of blood stasis syndrome [14].

In this work, we aim to use a comprehensive network pharmacology-based approach to investigate the mechanisms of how ZJW exerts the therapeutic effects on gastritis. The flowchart of the experimental procedures of our study was shown in Fig. 1.

\section{Methods}

\section{Data preparation}

\section{Chemical ingredients database building}

To determine the chemical ingredients of the two herbs contained in ZJW, we performed a search by Traditional Chinese Medicine Systems Pharmacology Database [15] (TCMSP, http://lsp.nwu.edu.cn/tcmsp.php, updated on May 31, 2014) and Chinese Academy of Sciences
Chemistry Database [16] (CASC, http://www.organc hem.csdb.cn/scdb/main/slogin.asp, updated on February $11,2018)$ and related literatures using " $R$. coptidis" and "E. rutaecarpa" as the queries. TCMSP is a unique systems pharmacology database of Chinese herbal medicines which captures the herbs, chemicals, targets and drug-target networks [17]. CASC, one of the most comprehensive chemical databases in the world, can provide the chemical information of traditional Chinese herbs and natural products [13].

\section{The prediction of known therapeutic targets acting on gastritis} We collected gastritis targets from two sources. One was the DrugBank database [18] (http://www.drugbank.ca/, version 4.3), which is a unique bioinformatics and cheminformatics resource that combines detailed drug data with comprehensive drug target information. The keyword "gastritis" was used and only drug-target interactions for drugs approved by the Food and Drug Administration (FDA) for treating gastritis and human gene/protein targets were selected. The other resource was the Online Mendelian Inheritance in Man (OMIM) database [19] (http://www.omim.org/, updated on May 4 , 2018). The OMIM database catalogued all known diseases with a genetic component and linked them to the relevant genes in the human genome and provided references for further research and tools for genomic analysis of a catalogued gene [19]. We searched the OMIM database with the query "gastritis" as well.

\section{The prediction of putative targets of the ingredients within ZJW}

The integrative efficacy of the ingredients in ZJW was determined by analyzing the ingredients and targets interactions obtained from TCMSP Database and STITCH DataBase [20] (http://stitch.embl.de/, ver. 4.0) with the species limited as "Homo sapiens". STITCH was a database to explore known and predicted interactions between chemicals and proteins. Only the proteins which had direct interactions with each chemical in ZJW were selected as the putative targets.

\section{Protein-protein interaction data}

Protein-protein interaction (PPI) data were derived from eight major existing public PPI databases, namely, String [21], Reactome [22], Online Predicted Human Interaction Database (OPHID) [23], InAct [24], Human Protein Reference Database (HPRD) [25], Molecular Interaction Database (MINT) [26], Database of Interacting Proteins (DIP) [27], and PDZBase [28]. The eight open databases covered the majority of known human protein-protein interactions information. Detailed information about these PPI databases was provided in Additional file 1: Table S1. All the data were merged after removing redundant entries and 


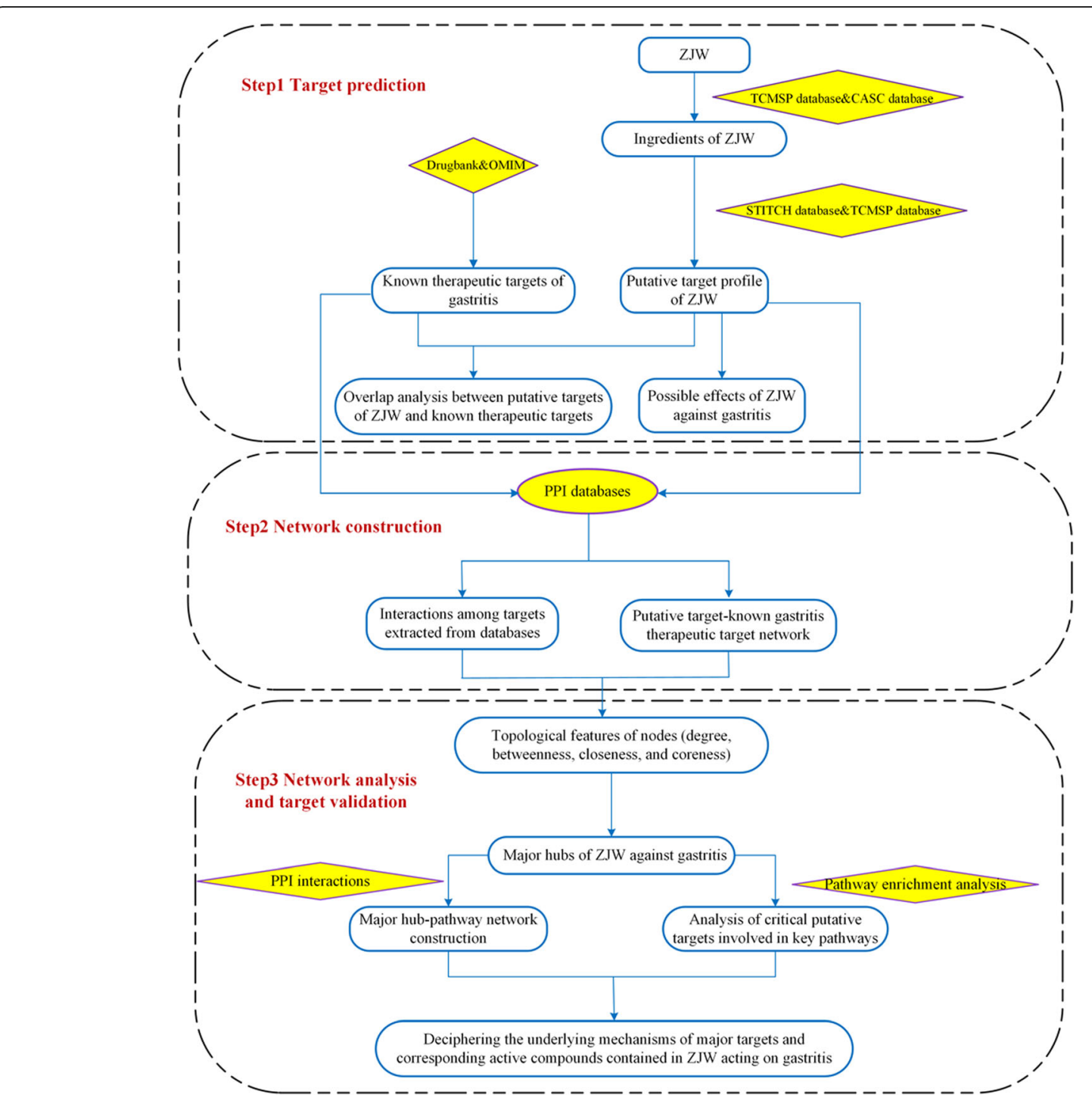

Fig. 1 The flowchart of network pharmacology-based strategy for deciphering the mechanisms of ZJW acting on gastritis. Abbreviations: ZJW, Zuojinwan; TCMSP, Traditional Chinese Medicine Systems Pharmacology; CASC, Chinese Academy of Sciences Chemistry; OMIM, Online Mendelian Inheritance in Man; PPI, protein-protein interaction

the differing ID types of the proteins were converted to UniProt IDs.

\section{Network construction}

The putative targets of ZJW, the gastritis disease targets and interactional proteins were connected based on the protein-protein interactions derived from the eight public databases. Then the interactions between proteins of the putative targets of ZJW, known therapeutic targets for gastritis and interactional human targets were combined to construct putative ZJW target-known therapeutic targets of gastritis network. It can be applied to illustrate the relationships between putative targets contained in ZJW and known therapeutic targets of gastritis. The graphical interactions in this network was visualized using Cytoscape software [29] (version 3.6.0, Boston, MA, USA). In addition, a null model was constructed to verify the significance of the putative ZJW target-known therapeutic targets of gastritis network.

\section{Putative ZJW target-known therapeutic targets of the gastritis network}

Based on the interactions between putative ZJW targets, known therapeutic targets of gastritis and interactional human proteins, the whole links of the network were established. According to previous reports [30-32], a node would be defined as a hub when the degree of the node was more than twofold the median degree of all the nodes in the same network. After selecting hubs from the network, the direct interactions among hubs 
was constructed. Next, we used four topological parameters, "degree", "betweenness", "closeness", and "coreness" to evaluate the topological importance of the selected hubs. The definitions for each topological parameter mentioned above are given in 'Definitions of topological propertie' section. Only the hubs whose topological parameters were greater than the corresponding median values were considered as major hubs.

\section{Definitions of topological properties}

As for each node $\mathrm{i}$ in the networks, the definitions of four measures for evaluating its topological properties were shown as follows. 'Degree' is defined as the number of links to node i. 'Node betweenness' represents the number of the shortest paths between pairs of nodes that ran through node i. The concept of 'Node closeness' is the inverse of the sum of distances from the node $i$ to all other nodes. The closeness centrality can also be seen as a metric of the time it will take to sequentially spread information from node $\mathrm{i}$ to all the other accessible nodes. We usually use 'Degree', 'Node betweenness' and 'Node closeness centralities' to describe a protein's topological importance in the network. In other words, the relationship between these three topological parameters and importance of corresponding protein is direct proportional [33]. 'Coreness' is shell index of 'K-core' decomposition. K-core analysis is an iterative procedure in which the nodes are removed from the networks in descending degree order. The highest degree node was selected as the main core or the highest $\mathrm{k}$-core of the network. After repeatedly deleting vertices from the network whose degree is less than $\mathrm{k}$, a k-core sub-network of the original network forms. This process generates a series of sub-networks that uncover the main hierarchical layers of the original network step by step. On this basis, 'Coreness' is a parameter to measure the centrality of node $\mathrm{i}$.

\section{Null model construction}

In order to validate the non-trivial structure of putative ZJW target-known therapeutic targets of the gastritis network, we constructed a null model in which all the nodes of the ZJW network were conserved as well as the number of links, but the nodes were rewired randomly in terms of the Erdös-Rényi model. We also used the corresponding topological parameters to screen out the major hubs in the null model.

\section{Pathway enrichment performance}

To cluster the biological functions of the major hubs, they were uploaded to MetaCore ${ }^{\mathrm{mm}}$ [34] (https://portal.g enego.com) as Gene Symbol style to run pathway enrichment analysis. MetaCore online database delivers high-quality biological systems content as well as essential data and analytics, including sophisticated integrated pathway and network analysis for multi-omics data. First the list of major hubs was input into the Metacore database as a new event, we selected the new event in the online database and clicked on the icons with "protein functional annotation and enrichment analysis" and "network construction and analysis" successively to obtain the main pathways and network distribution involved in the functional regulation. Then the results could be analyzed in the next step. Also, we calculated and evaluated significant pathways assisted by Database Visualization and Integrated Discovery system [35] (DAVID, http://david.abcc.ncifcrf.gov/home.jsp, version 6.7) and the Kyoto Encyclopedia of Genes and Genomes database [36] (KEGG, http://www.genome.jp/kegg/, updated on April 18, 2016). Then we compared the generated results with a null model to assess the statistical significance.

\section{Results}

Composite ingredients of ZJW

A total of 170 chemical ingredients (Additional file 2: Table S2) of the two herbal medicines in ZJW were retrieved from TCMSP and CASC and related literatures, including 29 ingredients in $R$. coptidis and 141 ingredients in E. rutaecarpa.

\section{Known therapeutic targets acting on gastritis}

In total, 75 known therapeutic targets for gastritis were collected from DrugBank database. And 15 known therapeutic targets for the treatment of gastritis were acquired based on OMIM database. After eliminating the redundancy, a total of 90 known therapeutic targets in the treatment of gastritis were collected in this study. The details were described in Additional file 3: Table S3.

\section{Putative targets for ZJW}

According to the target prediction system in TCMSP and STITCH databases, the quantity of putative targets for $R$. coptidis and E. rutaecarpa was 175 and 348, respectively. There were 106 putative targets of the two herbs overlapped, which was suggestive of potential interactions between $R$. coptidis and E. rutaecarpa in the course of treatment. Detailed information about putative targets is provided in Additional file 4: Table S4.

\section{Network and pathway analysis}

To shed light on the potential mechanisms of ZJW acting on gastritis, the putative ZJW target-gastritis related target network consisting of putative ZJW targets, known therapeutic target for gastritis and interactional human proteins was constructed based on PPI databases. As a result, the network was composed of 5559 nodes and 21567 edges. For detailed information about this network, see Additional file 5: Table S5. 
A hub target in a network is regarded as a crucial node and used to measure the essence of the whole network. It has been reported that [37], nodes will be defined as hubs if their degree is greater than twofold the median degree of all nodes in the network. In this network, we used the twofold median value of node degree (two) as a cutoff point primarily. After removing the targets whose degree was less than or equal to two, 2654 nodes were encoded as hubs. Then four topological features, "degree", "betweenness", "closeness", and "coreness", of selected hubs were calculated to screen the major hubs in the network. Only the hubs with higher values of "degree", "betweenness", "closeness", and "coreness" (above the median value of all the network nodes) were identified as the major hub targets. To focus on the further enrichment analysis of crucial hubs, we discarded some targets which might play unimportant roles in the network according to the topological features. Therefore, we kept proteins with "degree" > 4, "betweenness" > 0.0002 , "closeness" > 0.3919 and "coreness" $>5$ as major hubs. Eventually, 118 major hubs were picked out for further study, of which 59 targets were from putative targets of ZJW and 12 targets were derived from known therapeutic target of gastritis. The details were shown in Additional file 6: Table S6.

To clarify the biological actions of these hubs, a pathway enrichment analysis was performed based on Metacore database. We fed MetaCore a gene list of major hubs, generating relevant pathways which might have an important influence on the biological process for ZJW treating gastritis. Only pathways with $P$-value $<0.05$ were considered as significant pathways. The full list of significant pathways was shown in Additional file 7: Table S7. We analyzed the data and relevant biological processes, choosing top ten remarkable significant pathways according to the $P$ value for further study. The top ten main pathways were shown in Fig. 2.
Modularity could be an important aspect of a network. Nodes highly interconnected within a network were usually participated in the same biological modules. In terms of the functional distribution of major hubs and main pathways, the interaction network of putative ZJW targets and gastritis-related targets and interactional targets was divided into three modules. The assortments of the main pathways and modules are shown in Fig. 3. The maximum module consisting of major hubs was associated with inflammation suppression and immune responses, and the second module was sorted as a progression of growth and development, while the minimum module was concentrated in G-protein-coupled receptor signaling.

We analyzed the null-model-network topology and performed pathway enrichment analysis in the same way as we interpreted the ZJW network, and no modules or pathways were found since just a few genes without any interaction were screened out. The details of the whole null-model-network were shown in Additional file 8: Table S8. The major hubs of null-model- network were also supplemented in Additional file 9: Table S9. The null-model-network topology (Fig. 4a) and the core structure of the random network after calculation of four topological properties (Fig. 4b) were shown in Fig. 4. By contrast with the null model, the significance of ZJW network was verified.

Potential mechanisms of ZJW in the treatment of gastritis In the maximum module, cytokines and genes were enriched in inflammatory pathways including IL-11 signaling pathway via MEK/ERK and PI3K/AKT cascades, IL-18 signaling pathway, GM-CSF signaling pathway, histamine signaling in dendritic cells and gastrin in inflammatory response. Most of these pathways contain PI3K/AKT signaling pathway and NF-kB mediated signaling pathway, and both play important roles in

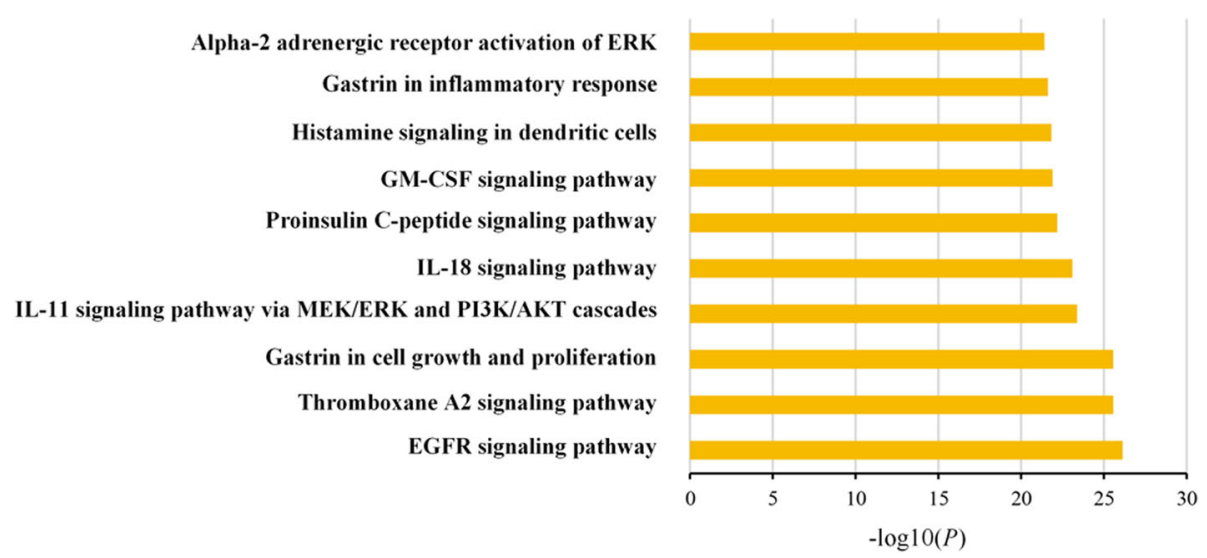

Fig. 2 Main pathways enriched by major hubs from Metacore database. The top 10 pathways measured by counts were selected to demonstrate the crucial biological actions of major hubs. The abscissa stands for target counts in each pathway; and the ordinate stands for main pathways 


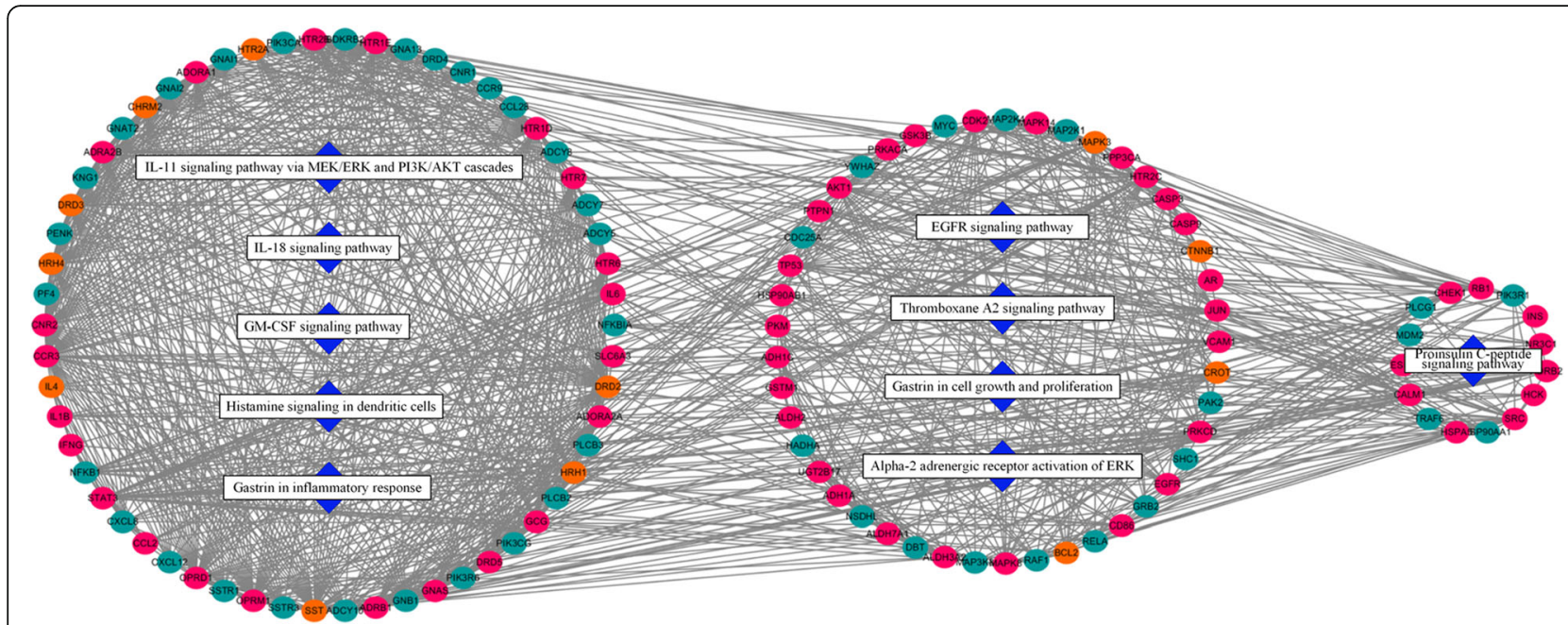

Fig. 3 The interaction network between major hubs and main pathways. Round red nodes stand for putative targets of ingredients contained in ZJW; round orange nodes stand for known therapeutic targets for gastritis; round green nodes stand for the main link targets between red nodes and orange nodes; blue diamonds stand for main pathways based on enrichment analysis of major hubs

inflammatory response as well as cell proliferation. Usually, the extracellular cytokine binding to its corresponding receptor leads to a series of activation of downstream molecules, and sequentially PI3K/AKT signaling pathway or NF- $\mathrm{kB}$ mediated signaling pathway can be activated, resulting in transcription of relevant transcription factors and secretion of some inflammatory factors participating in the process of inflammation.

EGFR signaling pathway occupied a leading position in the second module. The epidermal growth factor receptor (EGFR) is a cell surface protein that binds to epidermal growth factor. Binding of the protein to an extracellular growth factor ligand induces receptor dimerization and tyrosine autophosphorylation and leads to diverse biologic responses, including cell proliferation, differentiation, cell motility and survival [38]. EGFR dimerization and tyrosine autophosphorylation can activate several intracellular signaling pathways, such as PI3K/AKT signaling pathway, JUK signaling pathway. These pathways play an important role in cell growth and proliferation. As well, EGFR signaling pathway is partly involved in those main growth and development-related pathways including thromboxane A2 signaling pathway, gastrin in cell growth and proliferation, alpha-2 adrenergic receptor activation of ERK.

Proinsulin C-peptide signaling pathway composed a part of the minimum module. One of the putative receptor for proinsulin C-peptide was supposed to be a specific GPCR linked to the G-protein alpha-i family [39-41], which could stimulate PI3K/AKT and NF- $\mathrm{KB}$. And then transcription of inflammatory genes and anti-apoptotic genes was activated by proinsulin C-peptide-stimulated NF- $\kappa B$. A series of biological functions of inflammatory response and immune actions would be initiated. Furthermore, proinsulin C-peptide also participated in regulation ERK. And the binding of proinsulin C-peptide and GPCR triggered ERK signaling via PLC-beta-dependent stimulation of PKC or PI3K-dependent pathway [42]. These biological reactions contributed a lot to cell proliferation.

\section{Discussion}

Gastritis can be caused by various factors, and some remarkable alterations of the stomach mucosa including epithelial damage and mucosal inflammation will appear once patients suffer from gastritis [43]. NF- $\mathrm{kB}$ mediated signaling pathway is activated while gastritis occurs. $\mathrm{NF}-\mathrm{kB}$ can promote the expression of pro-inflammatory cytokines genes such as IL- $1 \beta$, TNF- $\alpha$, IL- $12 \beta$, IL-6, IL-8 [44-49]. These cytokines are attributed to the inflammation of gastric epithelial cells. It has been reported that the injury of gastric mucosa barrier has a close relationship with gastritis, and long-playing activation by inflammatory factors will induce the severe lesion in gastric glandular tissues when the gastric mucosa is not well regenerated [50]. EGF is one of the most important factors in gastric tissue repair and cell regeneration, showing its effects through the combination with its receptor (EGFR) located in gastric epithelial basal or bilateral membrane. The level of the expression of EGFR can be highly detected in the damaged gastric barrier and perfused epithelia while it is rarely found in normal epithelial cells. It is also found that EGFR expressed more highly in the surface mucosal layer than the deep and muscle layers of stomach, implying its effects on the promotion of the growth and recovery of damaged gastric epithelial cells [51]. However, excessive expression of EGFR can induce malignant growth in gastric epithelial cells and may cause canceration to some extent $[52,53]$. 


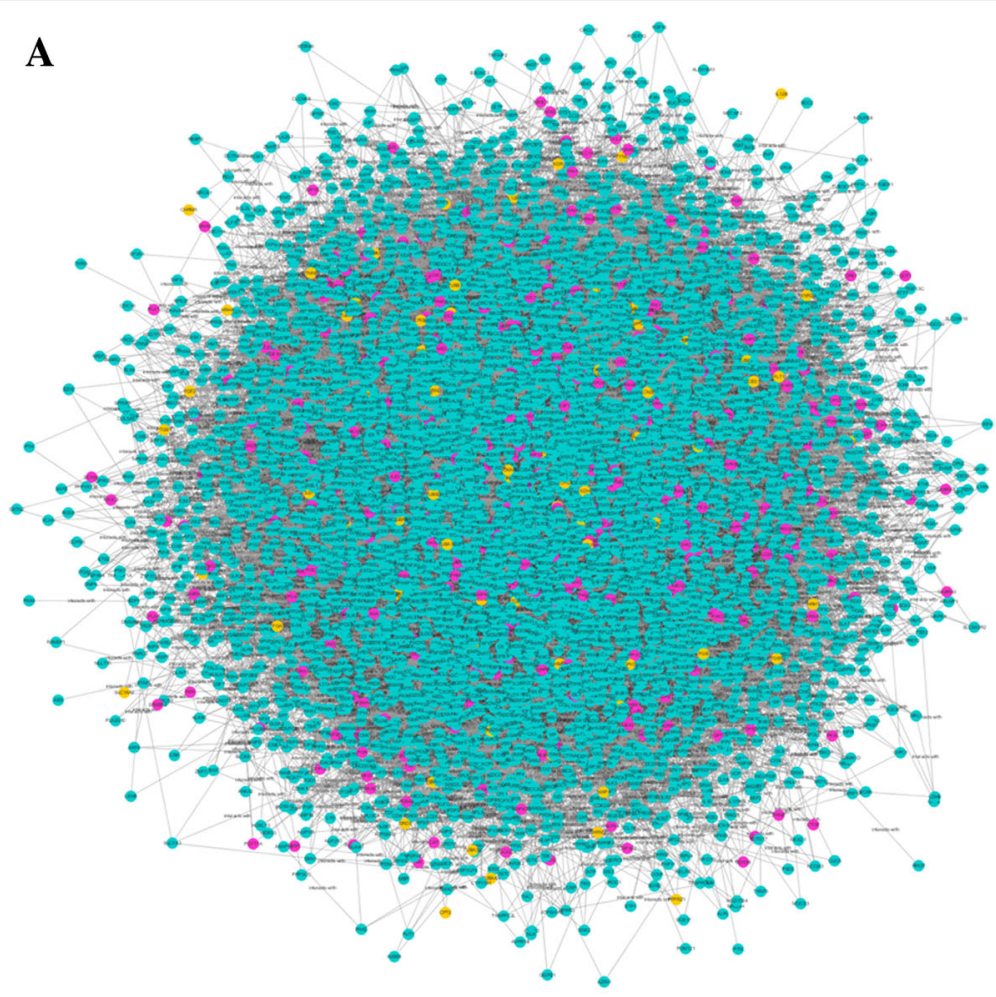

B

Fig. 4 Null model network. a The null model network composed of the same nodes and the number of edges from ZJW network but randomized links based on Erdös-Renyí model. $\mathbf{b}$ The core structure of null model network after calculation of four topological properties

Therefore, to regulate the balance of the expression of EGFR is very pivotal in gastric mucosa development and repairment. Besides, as a multifunctional gastro-intestinal hormone, gastrin is secreted by G cells in the gastric antrum and plays important roles in stimulating acid secretion, cell growth and mucous regeneration $[54,55]$. As a result of increase of serum concentration of gastrin accompanying with increased grades of atrophic gastritis, the serum gastrin level is taken as a significant biomarker for evaluating the status of gastric inflammation [56, 57]. It is also evident that gastrin is a growth factor which can induce gastric carcinogenesis $[58,59]$.

The pathological processes of gastritis are closely related to inflammatory response and cell development. Five putative targets of ZJW (interleukin-6[IL-6], IL-1 $\beta$, tumor necrosis factor $[\mathrm{TNF}]-\alpha$, monocyte chemotactic protein [MCP]-1 and EGFR), and two known therapeutic targets of gastritis (cholecystokinin-B receptors[CCKBR] and IL-12 $\beta$ ) and a link target between ZJW and disease NF-kB are shown to be the most active factors that participate in these key pathways, which are implied an important role in the occurrence and promotion of gastritis. The main biological processes are shown in Fig. 5 .

Cytokines and chemokines can be produced when a signaling cascade is activated during the progression of lesion in the stomach, inducing chronic inflammatory response. Gastric epithelium can secret a series of chemokines including interleukin (IL)- 6 and IL- $1 \beta$, which are chemotactic for neutrophils and mononuclear cells. With the recruitment of neutrophils and macrophages 


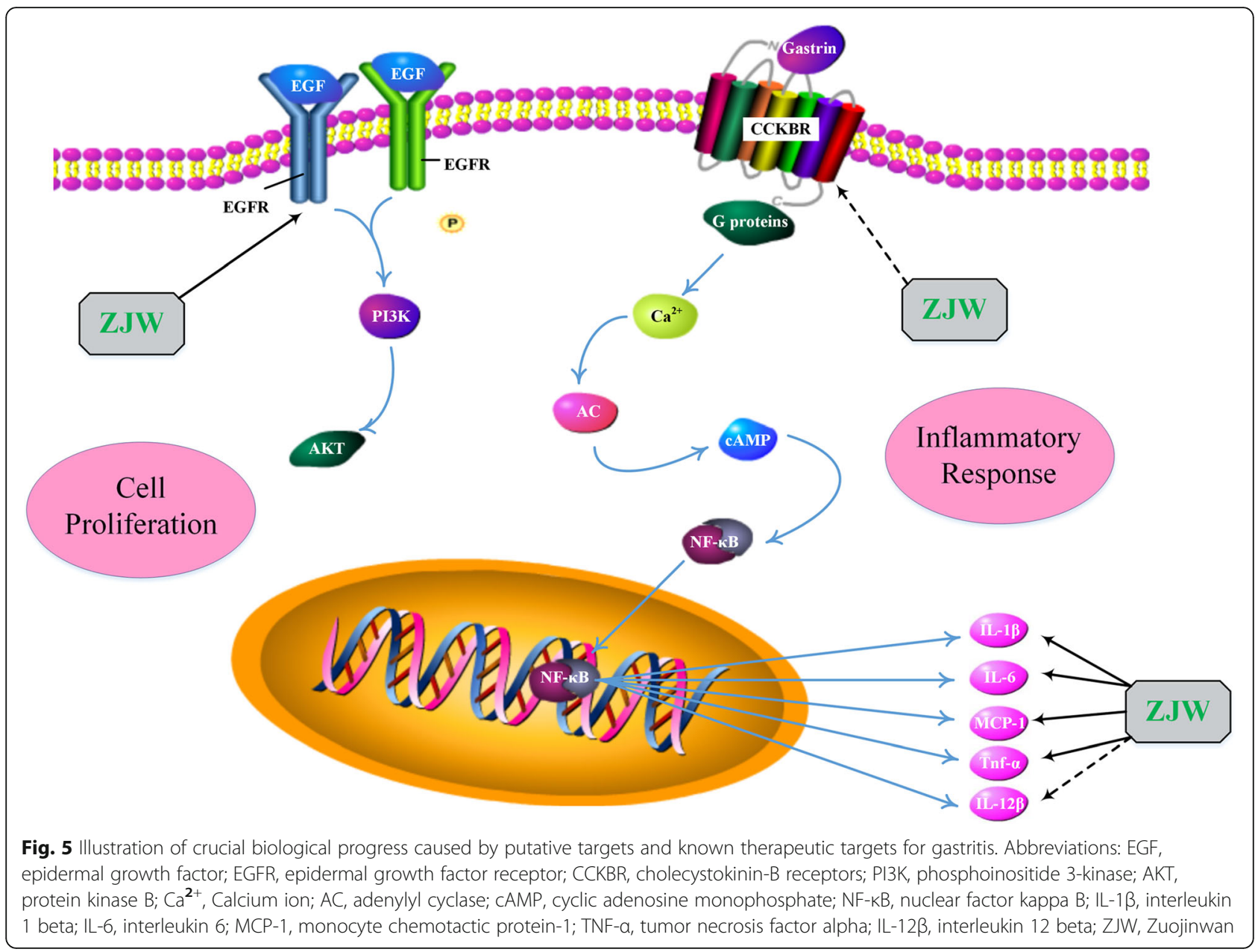

in the mucosa of stomach, the infiltration is the main factor of a chronic gastritis. Expect neutrophils and mononuclear cells, dendritic cells and $\mathrm{T}$ and $\mathrm{B}$ cells can also form infiltration and then activate the production of monocyte chemotactic protein (MCP)-1, tumor necrosis factor (TNF)- $\alpha$, IL-12 and so on [60]. IL-6 can be produced by $\mathrm{T}$ cells and macrophages that gives rise to immune response during infection or after injury which induces inflammation in the gastric mucosa or other tissues [61]. It is also a pleiotropic cytokine associated with the growth and differentiation of immunocyte as well as the expression of some other cytokines [62]. Furthermore, IL- 6 has been reported as a significant marker in the process of inflammation-related canceration [63, 64]. And there is evidence that in an inflammation-induced tumor model, IL-6 deficiency could relive the promotion of tumor development [65, 66]. MCP-1, known as CC-chemokine ligand 2 (CCL2), is a micromolecular potent chemoattractant for leukocytes to gather on the position where tissue is injured and inflammation or tumor are triggered $[60,67]$. Studies have demonstrated that many tumor cells can express CCL2, suggesting its role in the course of cancer [68-71]. It is also reported that tumor samples from 68 gastric cancer patients highly expressed CCL2, implied that CCL2 may have a close relationship with gastric disease. Thus, CCL2 is considered to be a predictive molecule for gastric carcinoma [72]. Secreted by monocytes and macrophages, TNF- $\alpha$ stimulates the production of a series of cytokine and play an important part in gastritis. TNF- $\alpha$ is suggested to be a considerable immune mediator in inflammatory response which is initiated by infection and other factors $[73,74]$. It has a positive influence on gastric mucosal apoptosis, and persistent apoptosis may cause extensive gastric mucosa damage like gastric ulcer $[75,76]$. IL- $1 \beta$ is referred to be a pro-inflammatory cytokine that can be largely produced as a result of host defense against external invasion and tissue lesions [77]. It is found that high expression of IL-1 $\beta$ in the stomach of transgenic mice can activate spontaneous severe gastric inflammatory response [78]. Therefore, IL-1 $\beta$ is an important signaling mediator in the promotion of gastritis. It is also emphasized on the investigation that the polymorphisms of IL1B gene may be involved in the 
high risk of the generation of gastric cancer [79-81]. Moreover, it is demonstrated that in a IL- $1 \beta$-deficient mice model, the infiltration of immune cells and gastric tumors are positively suppressed [82]. Gastrin is identified as the chief stimulant of gastric acid secretion which is produced by G cells in the gastric antrum [50]. Gastrin exerts its effects primarily through binding to cholecysto kinin-B receptors (CCKBR) on enterochromaffin-like (ECL) cells of the gastric mucosa [83, 84]. CCKBR, also known as cholecystokinin-2 receptors (CCK2R), is a seven transmembrane G-protein coupled receptor that is mostly expressed in gastric fundus [85]. The expression of gastrin-CCKBR indicates the atrophic changes in the stomach $[54,56]$. Consequently, gastrin-CCKBR level is a vital standard for measuring the severity of gastritis [57]. In addition, the expression of CCKBR is regarded as a symbol that has a close relationship with gastric canceration. IL-12 $\beta$ is a pro-inflammatory cytokine that is produced by various immune cells [86, 87]. The expression of IL-12 $\beta$ can be remarkably increased when the inflammatory response occurs in the layer of gastric tissues [88]. And studies have demonstrated that its regulatory gene IL12B is the target gene of NF- $\mathrm{KB}$ [89]. Importantly, the activation of NF- $\mathrm{kB}$ is critically responsible for the secretion of cytokines including IL-6, IL- $1 \beta$, TNF- $\alpha$ and so on [90]. It has been reported that the extract of ZJW could significantly reduce the activity of NF- $\mathrm{kB}$ as well as some pro-inflammatory cytokines [91]. As is reported in the previous studies, ZJW was found to be an effective inhibitor for IL-1 $\beta$, IL- 6 and TNF- $\alpha$ in a concentration -dependent manner in LPS-induced RAW 264.7 macrophages [92]. It is also validated that ZJW could relieve the inflammation by downregulating the levels of TNF- $\alpha$ and IL- $1 \beta$ in rats, reducing the expression of the gene TNFA and IL1B in gastric mucosa [93]. And reducing cytokine IL-6 is another effective way for ZJW to attenuate the infiltration of immune cells and injury area in the stomach of rats [94]. It is evident that ZJW can upregulate the expression level of EGFR in rats with gastric ulcer induced by acetic acid, promoting the restoration of damaged gastric mucosa [95]. The down-regulation of ZJW for MCP-1 has been inferred as a result of anti-inflammatory effects of ZJW. Moreover, ZJW may exert its therapeutic effects through inhibiting the expression of IL- $12 \beta$ and CCKBR, cutting down the opportunity of further deterioration of gastritis. In summary, blocking these pro-inflammatory factors and raising relevant growth factors may be the pharmacological mechanism of ZJW acting on gastritis.

Besides, stimulation of growth and development signaling (EGFR signaling pathway, Gastrin in cell growth and proliferation and so on) leads to cell growth and proliferation. Excessive or inappropriate cell growth and proliferation can be one of the major causes of tumor development $[96,97]$. It has been reported that excessive NF- $\mathrm{kB}$ activation was detected in human colorectal cancer tissues [98], and ZJW played a suppressive role in the expression of NF- $\mathrm{kB}$ [99]. Furthermore, an investigation concerning effects of ZJW on eight kinds of human cancer cell lines suggested that ZJW has significant anti-cancer activities due to up-regulation of pro-apoptosis proteins (caspase-3, caspase-9, Bax and Bak) and down-regulation of antiapoptosis proteins (Bcl-2 and Bcl-xl) [100]. However, anti-cancer effects of ZJW in vivo and specific molecular mechanisms deserved to be deeply explored.

In addition, among the putative targets of ZJW, IL-6 is putative target of 2-Methoxy-4-vinylphenol and caffeine from E. rutaecarpa, IL-1 $\beta$ is putative target of alpha-humulene from E. rutaecarpa, TNF- $\alpha$ is putative target of hexanal, palmitic acid, rutaecarpine from $E$. rutaecarpa and quercetin from $R$. coptidis, EGFR is putative target of rutin from $E$. rutaecarpa, MCP-1 is putative target of quercetin from $R$. coptidis. These compounds are proposed to be the active constituents of ZJW in the treatment of gastritis.

However, there are also some limitations for the use of network pharmacology approach to predict active ingredients and potential mechanisms. (i) the active ingredients screened might be inconsistent with the ingredients actually absorbed in blood of the patients with gastritis; (ii) to distinguish inhibitory effects from activated effects of the targets could be a difficult problem; (iii) the predicted results might be impacted by possible biases to highly studied pathways/functions. Therefore, further experimental verification of the potential effective ingredients is demanded to validate theoretical predictions.

\section{Conclusion}

TCM, one of the most important parts of complementary and alternative medicine, markedly contributes to the therapeutic action of gastrointestinal diseases. This study uses a scientific approach to holistically decipher that the pharmacological mechanisms of ZJW in the treatment of gastritis may be associated with its involvement into inflammation suppression and immune responses, growth and development pathways. Among these crucial biological functions, eight targets were identified as key active factors involved in the related pathways. However, to enhance the reliability of the results, further experimental experiments were demanded to validate these hypotheses.

\section{Additional files}

Additional file 1: Table S1. Detailed information on eight existing protein-protein interaction databases. (XLSX 12 kb) 
Additional file 2: Table S2. Ingredients of each herb contained in ZJW. (XLSX $1869 \mathrm{~kb})$

Additional file 3: Table S3. Known therapeutic targets for gastritis. (XLSX $14 \mathrm{~kb}$ )

Additional file 4: Table S4. Putative targets of each herb contained in ZJW and overlap between the two herbs. (XLSX 38 kb)

Additional file 5: Table S5. Interactions between putative targets of ZJW and known therapeutic target for gastritis. (XLSX $474 \mathrm{~kb}$ )

Additional file 6: Table S6. Major hubs in the network. (XLSX 14 kb)

Additional file 7: Table S7. Significant pathways. (XLSX $18 \mathrm{~kb}$ )

Additional file 8: Table $\mathbf{8 8}$. The whole null model according to Erdös-Rényi model. (XLSX 340 kb)

Additional file 9: Table S9. The major hubs of null-model- network. (XLSX $8 \mathrm{~kb})$

\section{Abbreviations}

AC: Adenylyl cyclase; AKT: Protein kinase B; $\mathrm{Ca}^{2+}$ : Calcium ion; CAMP: Cyclic adenosine monophosphate; CASC: Chinese Academy of Sciences Chemistry; CCKBR: Cholecystokinin-B receptors; CCL2: CC-chemokine ligand 2;

EGF: Epidermal growth factor; EGFR: Epidermal growth factor receptor; IL 12 $\beta$ : Interleukin 12 beta; IL-1 $\beta$ : Interleukin 1 beta; IL-6: Interleukin 6; MCP1: Monocyte chemotactic protein-1; NF-kB: Nuclear factor kappa B; OMIM: Online Mendelian Inheritance in Man; PI3K: Phosphoinositide 3-kinase; PPI: Protein-protein interaction; TCMSP: Traditional Chinese Medicine Systems Pharmacology; TNF-a: Tumor necrosis factor alpha; ZJW: Zuojinwan

\section{Acknowledgments}

We would like to thank Zhiqiang Luo for image processing and Tao Lu for manuscript review in this research.

\section{Funding}

This study was supported by the Start-up fund from Beijing University of Chinese Medicine to Yuanyuan Shi (No.1000061020013).

\section{Availability of data and materials}

The datasets used and/or analyzed during the current study are available from the corresponding author on reasonable request.

\section{Authors' contributions}

SYY conceived and designed the experiments; YGH, WWB and WX performed the experiments and wrote the paper; $X M, Z L L, D L$ and $G R$ analyzed the data. All authors have read and approved the final manuscript.

\section{Ethics approval and consent to participate}

Not applicable.

\section{Consent for publication}

Not applicable.

\section{Competing interests}

The authors declare that they have no competing interests.

\section{Publisher's Note}

Springer Nature remains neutral with regard to jurisdictional claims in published maps and institutional affiliations.

Received: 19 June 2018 Accepted: 18 October 2018 Published online: 01 November 2018

\section{References}

1. Paduraru G, Lupu W, Diaconescu S, Burlea M. Particular considerations on helicobacter pylori-associated chronic gastritis in child. Romanian J Pediatr. 2011;60(1):29-33.

2. Ali El Zahaby A, Abdel Alim A, Elsharawy A. Role of Rebamipide and $\backslash$ or pantoprazole in preventing dexamethasone induced gastritis in senile male albino rats. Egypt J Hosp Med. 2017;67(2):789-505.
3. Jones L, Brooks D. Cash. Gastritis. In Digestive Disease, National Institute of Diabetes and Digestive and Kidney Diseases. https://www.niddk.nih.gov/ health-information/digestive-diseases/gastritis. Accessed July 2015.

4. Rosen \& Barkin's 5-Minute Emergency Medicine Consult (4 ed.). Lippincott Williams \& Wilkins. 2015:447.

5. Ferri's Clinical Advisor 2013,5 Books in 1, Expert Consult - Online and Print,1: Ferri's Clinical Advisor 2013. Elsevier Health Sciences. 2016:417.

6. Li B, Xue X, Xia W, Hua Y, Li X, Tao W, et al. A systems biology approach to understanding the mechanisms of action of Chinese herbs for treatment of cardiovascular disease. Int J Mol Sci. 2012;13(10):13501-20.

7. Zhao F, Guochun L, Yang Y, Shi L, Xu L, Yin L. A network pharmacology approach to determine active ingredients and rationality of herb combinations of modified-Simiaowan for treatment of gout. J Ethnopharmacol. 2015;168:1-16.

8. Fang J, Wang L, Wu T, Yang C, Gao L, Cai H, et al. Network pharmacologybased study on the mechanism of action for herbal medicines in Alzheimer treatment. J Ethnopharmacol. 2016:196:281-92.

9. Rui Y, Yin W, Shen W, Liu Y, Xin D. Comparative pharmacokinetics of dehydroevodiamine and coptisine in rat plasma after oral administration of single herbs and Zuojinwan prescription. Fitoterapia. 2011;82(8):1152-9.

10. Zhao FR, Mao HP, Zhang H, Hu LM, Wang H, Wang YF, et al. Antagonistic effects of two herbs in Zuojin wan, a traditional Chinese medicine formula, on catecholamine secretion in bovine adrenal medullary cells. Phytomedicine. 2010;17(9):659-68.

11. Ming L, Yan CL, Liu HX, Wang TY, Shi XH, Liu JP, et al. Network pharmacology exploration reveals endothelial inflammation as a common mechanism for stroke and coronary artery disease treatment of Danhong injection. Sci Rep. 2017;7(1):15427.

12. Fang $H Y$, Zeng HW, Lin LM, Chen $X$, Shen XN, Fu P, et al. A network-based method for mechanistic investigation of Shexiang Baoxin Pill's treatment of cardiovascular diseases. Sci Rep. 2017;7:43632.

13. Yu G, Zhang Y, Ren W, Dong L, Li J, Geng Y, et al. Network pharmacologybased identification of key pharmacological pathways of yin-Huang-QingFei capsule acting on chronic bronchitis. Int J Chron Obstruct Pulmon Dis. 2017;2:85-94

14. Yue SJ, Xin LT, Fan YC, Li SJ, Tang YP, Duan JA, et al. Herb pair DangguiHonghua: mechanisms underlying blood stasis syndrome by system pharmacology approach. Sci Rep. 2017:7:40318.

15. Wang $\mathrm{CH}$, Zhong $Y$, Zhang Y, Liu JP, Wang YF, Jia WN, et al. A network analysis of the Chinese medicine Lianhua-Qingwen formula to identify its main effective components. Mol BioSyst. 2015;12(2):606-13.

16. Wang SS, Xu HY, Yan M, Wang XG, Shi Y, Huang B, et al. Characterization and rapid identification of chemical constituents of NaoXinTong capsules by UHPLC-linear ion trap/Orbitrap mass spectrometry. J Pharm Biomed Anal. 2015:111:104-18

17. Xu T, Li S, Sun Y, Pi Z, Liu S, Song F, et al. Systematically characterize the absorbed effective substances of Wutou decoction and their metabolic pathways in rat plasma using UHPLC-Q-TOF-MS combined with a target network pharmacological analysis. J Pharm Biomed Anal. 2017:141:95-107.

18. Sheng S, Wang J, Wang L, Liu H, Li P, Liu M, et al. Network pharmacology analyses of the antithrombotic pharmacological mechanism of Fufang Xueshuantong capsule with experimental support using disseminated intravascular coagulation rats. J Ethnopharmacol. 2014;154(3):735-44.

19. Hamosh A, Scott AF, Amberger J, Bocchini C, Valle D, Mckusick VA. Online Mendelian inheritance in man (OMIM), a knowledgebase of human genes and genetic disorders. Nucleic Acids Res. 2005;33(1):514-7.

20. Hong M, Li S, Tan HY, Cheung F, Wang N, Huang J, et al. A network-based pharmacology study of the herb-induced liver injury potential of traditional Hepatoprotective Chinese herbal medicines. Molecules. 2017;22(4):632.

21. Hsia CW, Ho MY, Shui HA, Tsai CB, Tseng MJ. Analysis of dermal papilla cell interactome using STRING database to profile the ex vivo hair growth inhibition effect of a vinca alkaloid drug, colchicine. Int J Mol Sci. 2015:16(2):3579-98.

22. D'Eustachio P. Reactome knowledgebase of human biological pathways and processes. Methods Mol Biol. 2011:694:49-61.

23. Brown KR, Jurisica I. Online predicted human interaction database. Bioinformatics. 2005;21(9):2076-82.

24. Kerrien S, Aranda B, Breuza L, Bridge A, Broackes-Carter F, Chen C, et al. The IntAct molecular interaction database in 2012. Nucleic Acids Res. 2012;40:841-6.

25. Prasad TSK, Kandasamy K, Pandey A. Human protein reference database and human Proteinpedia as discovery tools for systems biology. Methods $\mathrm{Mol}$ Biol. 2009;577:67-79. 
26. Licata L, Briganti L, Peluso D, Perfetto L, lannuccelli M, Galeota E, et al. MINT, the molecular interaction database: 2012 update. Nucleic Acids Res. 2012;40:857-61.

27. Lehne B, Schlitt T. Protein-protein interaction databases: keeping up with growing interactomes. Hum Genomics. 2009;3(3):1-7.

28. Beuming T, Skrabanek L, Niv MY, Mukherjee P, Weinstein H. PDZBase: a protein-protein interaction database for PDZ-domains. Bioinformatics. 2005; 21(6):827-8

29. Shannon P, Markiel A, Ozier O, Baliga NS, Wang JT, Ramage D, et al. Cytoscape: a software environment for integrated models of biomolecular interaction networks. Genome Res. 2003;13(11):2498-504.

30. Guo Q, Zhong M, Xu H, Mao X, Zhang Y, Lin N. A systems biology perspective on the molecular mechanisms underlying the therapeutic effects of Buyang Huanwu decoction on ischemic stroke. Rejuvenation Res. 2015;18(4):313-25.

31. Zhang Y, Wang D, Tan S, Xu H, Liu C, Lin N. A systems biology-based investigation into the pharmacological mechanisms of wu tou tang acting on rheumatoid arthritis by integrating network analysis. Evid Based Complement Alternat Med. 2013;2013(1):548498.

32. Zhang Y, Guo X, Wang D, Li RS, Li XJ, Xu Y, et al. A systems biology-based investigation into the therapeutic effects of Gansui Banxia tang on reversing the imbalanced network of hepatocellular carcinoma. Sci Rep. 2014;4(2):4154.

33. Wang L, Tan N, Hu J, Wang H, Duan D, Ma L, et al. Analysis of the main active ingredients and bioactivities of essential oil from Osmanthus fragrans Var. thunbergii using a complex network approach. BMC Syst Biol. 2017;11(1):144.

34. Yang ES, Willey CD, Mehta A, Crowley MR, Crossman DK, Chen D, et al. Kinase analysis of penile squamous cell carcinoma on multiple platforms to identify potential therapeutic targets. Oncotarget. 2017;8(13):21710-8.

35. Dennis G, Sherman BT, Hosack DA, Yang J, Gao W, Lane HC, et al. DAVID: database for annotation, visualization, and integrated discovery. Genome Biol. 2003:4(5):3.

36. Research NA. KEGG: Kyoto encyclopedia of genes and genomes. Nucleic Acids Res. 1999;27(1):29-34.

37. Li S, Zhang ZQ, Wu L, Zhang XG, Li YD, Wang YY. Understanding ZHENG in traditional Chinese medicine in the context of neuro-endocrine-immune network. IET Syst Biol. 2007;1(1):51-60.

38. Marmor MD, Skaria KB, Yarden Y. Signal transduction and oncogenesis by ErbB/HER receptors. Int J Radiat Oncol Biol Phys. 2004;58(3):903-13.

39. Rigler $R$, Pramanik A, Jonasson P, Kratz G, Jansson OT, Nygren PA, et al. Specific binding of proinsulin C-peptide to human cell membranes. Proc Natl Acad Sci U S A. 1999;96(23):13318-23.

40. Zhong Z, Davidescu A, Ehrén I, Ekberg K, Jörnvall H, Wahren J, et al. Cpeptide stimulates ERK1/2 and JNK MAP kinases via activation of protein kinase C in human renal tubular cells. Diabetologia. 2005;48(1):187-97.

41. Al-Rasheed NM, Willars GB, Brunskill NJ. C-peptide signals via Galpha i to protect against TNF-alpha-mediated apoptosis of opossum kidney proximal tubular cells. J Am Soc Nephrol. 2006;17(4):986-95.

42. Kitamura T, Kimura K, Jung BD, Makondo K, Okamoto S, Cañas X, et al. Proinsulin C-peptide rapidly stimulates mitogen-activated protein kinases in Swiss $3 T 3$ fibroblasts: requirement of protein kinase C, phosphoinositide 3kinase and pertussis toxin-sensitive G-protein. Biochem J. 2001;355(Pt 1):123-9.

43. Sgouros SN, Bergele C. Clinical outcome of patients with helicobacter pylori infection: the bug, the host, or the environment? Postgrad Med J. 2006; 82(967):338-42.

44. Alzahrani S, Lina TT, Gonzalez J, Pinchuk IV, Beswick EJ, Reyes VE. Effect of helicobacter pylori on gastric epithelial cells. World J Gastroenterol. 2014; 20(36):12767-80

45. Posselt G, Backert S, Wessler S. The functional interplay of helicobacter pylori factors with gastric epithelial cells induces a multi-step process in pathogenesis. Cell Commun Signal. 2013;11:77.

46. Trepicchio WL, Wang L, Bozza M, Dorner AJ. IL-11 regulates macrophage effector function through the inhibition of nuclear factor-kappaB. J Immunol. 1997;159(11):5661-70.

47. Zimmerman MA, Selzman CH, Reznikov LL, Raeburn CD, Barsness K, Jr MIR, et al. Interleukin-11 attenuates human vascular smooth muscle cell proliferation. Ajp Heart Circ Physiol. 2002;283(1):175-80

48. Trepicchio WL, Dorner AJ. Interleukin-11: A gp130 Cytokine. Ann N Y Acad Sci. 2010;856(1):12-21.

49. Yan D, Kc R, Chen D, Xiao G, Im HJ. Bovine lactoferricin-induced antiinflammation is, in part, via up-regulation of interleukin-11 by secondary activation of STAT3 in human articular cartilage. J Biol Chem. 2013;288(44): 31655-69.
50. Wang LJ, Chen SJ, Chen Z, Cai JT, Si JM. Morphological and pathologic changes of experimental chronic atrophic gastritis (CAG)and the regulating mechanism of protein expression in rats. J Zhejiang Univ Sci B(Biomedicine \& Biotechnology). 2006;7(8):634-40.

51. Ichikawa $T$, Endoh $H$, Hotta $K$, Ishihara K. The mucin biosynthesis stimulated by epidermal growth factor occurs in surface mucus cells, but not in gland mucus cells, of rat stomach. Life Sci. 2000;7(9):1095-101.

52. Kopp R, Ruge M, Rothbauer E, Cramer C, Kraemling HJ, Wiebeck B, et al. Impact of epidermal growth factor (EGF) radioreceptor analysis on longterm survival of gastric cancer patients. Anticancer Res. 2002;22(2B):1161-7.

53. Wang $Y L$, Sheu BS, Yang HB, Lin PW, Chang YC. Overexpression of c-erb-B2 proteins in tumor and non-tumor parts of gastric adenocarcinoma-emphasis on its relation to $\mathrm{H}$. pylori infection and clinicohistological characteristics. Hepato-Gastroenterology. 2002;49(46):1172-6.

54. Maddalo G, Spolverato Y, Rugge M, Farinati F. Gastrin: from pathophysiology to cancer prevention and treatment. Eur J Cancer Prev. 2014;23(4):258-63.

55. Shimoyama T, Chinda D, Matsuzaka M, Takahashi I, Nakaji S, Fukuda S. Decrease of serum level of gastrin in healthy Japanese adults by the change of helicobacter pylori infection. J Gastroenterol Hepatol. 2014;29(S4): 25-8.

56. Naito $Y$, Ito $M$, Watanabe $T$, Suzuki $H$. Biomarkers in patients with gastric inflammation: a systematic review. Digestion. 2005;72(2-3):164.

57. Ito M, Haruma K, Kaya S, Kamada T, Kim S, Sasaki A, et al. Serological comparison of serum pepsinogen and anti-parietal cell antibody levels between Japanese and German patients. Eur J Gastroenterol Hepatol. 2002; 14(2):123-7.

58. Henwood M, Clarke PA, Smith AM, Watson SA. Expression of gastrin in developing gastric adenocarcinoma. Br J Surg. 2001;88(4):564-8.

59. Watson SA, Grabowska AM, Elzaatari M, Takhar A. Gastrin - active participant or bystander in gastric carcinogenesis? Nat Rev Cancer. 2006;6(12):936-46.

60. Sun X, Glynn DJ, Hodson LJ, Huo C, Britt K, Thompson EW, et al. CCL2-driven inflammation increases mammary gland stromal density and cancer susceptibility in a transgenic mouse model. Breast Cancer Res Bcr. 2017:19(1):4.

61. Poll TVD, Keogh CV, Guirao X, Buurman WA, Kopf M, Lowry SF. Interleukin-6 gene-deficient mice show impaired defense against pneumococcal pneumonia. J Infect Dis. 1997;176(2):439-44.

62. Cohen T, Nahari D, Cerem LW, Neufeld G, Levi BZ. Interleukin 6 induces the expression of vascular endothelial growth factor. J Biol Chem. 1996;271(2): 736-41.

63. Hong DS, Angelo LS, Kurzrock R. Interleukin-6 and its receptor in cancer: implications for translational therapeutics. Cancer. 2007;110(9):1911-28.

64. Thong-Ngam D, Tangkijvanich P, Lerknimitr R, Mahachai V, Theamboonlers A, Yong P. Diagnostic role of serum interleukin-18 in gastric cancer patients. World J Gastroenterol. 2006;12(28):4473-7.

65. Grivennikov S, Karin E, Terzic J, Mucida D, Yu GY, Vallabhapurapu S, et al. IL6 and STAT-3 are required for survival of intestinal epithelial cells and development of colitis-associated Cancer. Cancer Cell. 2009;15(2):103-13.

66. Bollrath J, Phesse TJ, Burstin VAV, Putoczki T, Bennecke M, Bateman T, et al. gp130-mediated Stat3 activation in enterocytes regulates cell survival and cell-cycle progression during colitis-associated tumorigenesis. Cancer Cell. 2009;15(2):91-102

67. Deshmane SL, Kremlev S, Amini S, Sawaya BE. Monocyte chemoattractant Protein-1 (MCP-1): an overview. J Interf Cytokine Res. 2009;29(6):313-26.

68. Wang H, Zhang L, Zhang IY, Chen X, Fonseca AD, Wu S, et al. S100B promotes glioma growth through Chemoattraction of myeloid-derived macrophages. Clin Cancer Res Official J Am Assoc Cancer Res. 2013;19(14): 3764-75.

69. Harlin H, Meng Y, Peterson AC, Zha Y, Tretiakova M, Slingluff C, et al. Chemokine expression in melanoma metastases associated with CD8+ Tcell recruitment. Cancer Res. 2009;69(7):3077-85.

70. Arnold JM, Huggard PR, Cummings M, Ramm GA, Chenevixtrench G. Reduced expression of chemokine (C-C motif) ligand-2 (CCL2) in ovarian adenocarcinoma. Br J Cancer. 2005;92(11):2024-31.

71. Kirk PS, Koreckij T, Nguyen HM, Brown LG, Snyder LA, Vessella RL, et al. Inhibition of CCL2 signaling in combination with docetaxel treatment has profound inhibitory effects on prostate cancer growth in bone. Int J Mol Sci. 2013:14(5):10483-96.

72. Tao LL, Shi SJ, Chen LB, Huang GC. Expression of monocyte chemotactic protein-1/CCL2 in gastric cancer and its relationship with tumor hypoxia. World J Gastroenterol. 2014;20(15):4421-7. 
73. Essadik A, Jouhadi H, Rhouda T, Nadifiyine S, Kettani A, Maachi F. Polymorphisms of tumor necrosis factor alpha in Moroccan patients with gastric pathology: new single-nucleotide polymorphisms in TNF-a-193 (G) a). Mediat Inflamm. 2015;2015:143941.

74. Takeuchi T, Miura S, Lin W, Uehara K, Mizumori M, Kishikawa H, et al. Nuclear factor-kappaB and TNF-alpha mediate gastric ulceration induced by phorbol myristate acetate. Dig Dis Sci. 2002;47(9):2070-8.

75. Nagata H, Akiba Y, Suzuki H, Okano H, Hibi T. Expression of Musashi-1 in the rat stomach and changes during mucosal injury and restitution. FEBS Lett. 2006:580(1):27-33.

76. Nakashita M, Suzuki H, Miura S, Taki T, Uehara K, Mizushima T, et al. Attenuation of acetic acid-induced gastric ulcer formation in rats by glucosylceramide synthase inhibitors. Dig Dis Sci. 2013;58(2):354

77. Dinarello CA. Biologic basis for interleukin-1 in disease. Blood. 1996;87(6): 2095-147.

78. Tu S, Bhagat G, Cui G, Takaishi S, Kurt-Jones EA, Rickman B, et al. Overexpression of interleukin-1beta induces gastric inflammation and cancer and mobilizes myeloid-derived suppressor cells in mice. Cancer Cell. 2008;14(5):408-19.

79. Yang J, Hu Z, Xu Y, Shen J, Niu J, Hu X, et al. Interleukin-1B gene promoter variants are associated with an increased risk of gastric cancer in a Chinese population. Cancer Lett. 2004;215(2):191-8.

80. Palli D, Saieva C, Luzzi I, Masala G, Topa S, Sera F, et al. Interleukin-1 gene polymorphisms and gastric cancer risk in a high-risk Italian population. Am J Gastroenterol. 2005;100(9):1941-8.

81. Kumar S, Kumar A, Dixit VK. Evidences showing association of interleukin-1B polymorphisms with increased risk of gastric cancer in an Indian population. Biochem Biophys Res Commun. 2009;387(3):456-60.

82. Shigematsu Y, Niwa T, Rehnberg E, Toyoda T, Yoshida S, Mori A, et al. Interleukin-1 $\beta$ induced by helicobacter pylori infection enhances mouse gastric carcinogenesis. Cancer Lett. 2013;340(1):141-7.

83. Dufresne M, Seva C, Fourmy D. Cholecystokinin and gastrin receptors. Physiol Rev. 2006;86(3):805-47.

84. Kopin AS, Lee YM, McBride EW, Miller LJ, Lu M, Lin HY, et al. Expression cloning and characterization of the canine parietal cell gastrin receptor. Proc Natl Acad Sci U S A. 1992;89(8):3605-9.

85. Mjønes P, Nordrum IS, Sørdal $\varnothing$, Sagatun L, Fossmark R, Sandvik A, et al. Expression of the cholecystokinin-B receptor in neoplastic gastric cells. Hormones Cancer. 2018;9(1):40-54.

86. Petkevicius $V$, Salteniene $V$, Juzenas $S$, Wex T, Link $A$, Leja $M$, et al. Polymorphisms of microRNA target genes IL12B, INSR, CCND1 and IL10 in gastric cancer. World J Gastroenterol. 2017;23(19):3480-7.

87. Pellicanò A, Sebkova L, Monteleone G, Guarnieri G, Imeneo M, Pallone F, et al. Interleukin-12 drives the Th1 signaling pathway in helicobacter pyloriinfected human gastric mucosa. Infect Immun. 2007;75(4):1738-44.

88. Guiney DG, Hasegawa P, Cole SP. Helicobacter pylori preferentially induces interleukin 12 (IL-12) rather than IL-6 or IL-10 in human dendritic cells. Infect Immun. 2003;71(7):4163-6.

89. Staff TPO. The NOD-like receptor signalling pathway in helicobacter pylori infection and related gastric cancer: a case-control study and gene expression analyses. PLoS One. 2014;9(6):e98899.

90. Yin Y, Si XL, Gao Y, Gao L, Wang J. The nuclear factor-kB correlates with increased expression of interleukin- 6 and promotes progression of gastric carcinoma. Oncol Rep. 2013;29(1):34-8.

91. Wang QS, Cui YL, Ding SL. Study on anti-inflammatory and antidepressant mechanisms of Zuojin pill ethanol extracts (ZIP). China Pharma Conf. 2011:10-9.

92. Wang QS, Cui YL, Dong TJ, Zhang XF, Lin KM. Ethanol extract from a Chinese herbal formula, "Zuojin pill", inhibit the expression of inflammatory mediators in lipopolysaccharide-stimulated RAW 264.7 mouse macrophages. J Ethnopharmacol. 2012;141(1):377-85.

93. Wang QS, Zhu XN, Jiang HL, Wang GF, Cui YL. Protective effects of alginatechitosan microspheres loaded with alkaloids from Coptis chinensis Franch. and Evodia rutaecarpa (Juss.) Benth. (Zuojin Pill) against ethanol-induced acute gastric mucosal injury in rats. Drug Design Dev Ther. 2015;9:6151-65.

94. Zhou XY, Yue H, Li CY, et al. Effect of Zuojin pill on inflammatory factors, oxidative stress factors and apoptosis factors in rats with stomach heat syndrome. Pharmacy Clin Chinese Materia Medica. 2017;8(2):49-52.

95. Pan Y, Ran R, Weng K, Chen YJ, Zhou FF, Yu HF. Therapeutic effects of Zuojinwan and its ingredients on rat gastric ulcer induced by acetic acid and influence on EGFR expression of the gastric mucous. Chinese J Integr Tradit West Med Digestion. 2008;16(6):368-71.
96. Lo HW, Hsu SC, Xia W, Cao X, Shih JY, Wei Y, et al. Epidermal growth factor receptor cooperates with signal transducer and activator of transcription 3 to induce epithelial-mesenchymal transition in Cancer cells via upregulation of TWIST gene expression. Cancer Res. 2007;67(19):9066-76.

97. Sharon B, Stuart T, Elizabeth B, Suzanne R, Filippo P, Izabela SK, et al. Bypassing cellular EGF receptor dependence through epithelial-tomesenchymal-like transitions. Clin Exp Metastasis. 2008;25(6):685-93.

98. Hien TT, Kim HG, Han EH, Kang KW, Jeong HG. Molecular mechanism of suppression of MDR1 by puerarin from Pueraria lobata via NF-kappaB pathway and CAMP-responsive element transcriptional activity-dependent up-regulation of AMP-activated protein kinase in breast cancer MCF-7/adr cells. Mol Nutr Food Res. 2010;54(7):918-28.

99. Sui H, Pan SF, Feng $Y$, Jin BH, Liu X, Zhou LH, et al. Zuo Jin wan reverses Pgp-mediated drug-resistance by inhibiting activation of the PI3K/Akt/NF-KB pathway. BMC Complement Altern Med. 2014;14(1):279.

100. Xu L, Qi Y, Lv L, Xu Y, Zheng L, Yin L, et al. In vitro anti-proliferative effects of Zuojinwan on eight kinds of human cancer cell lines. Cytotechnology. 2014;66(1):37-50.

\section{Ready to submit your research? Choose BMC and benefit from:}

- fast, convenient online submission

- thorough peer review by experienced researchers in your field

- rapid publication on acceptance

- support for research data, including large and complex data types

- gold Open Access which fosters wider collaboration and increased citations

- maximum visibility for your research: over $100 \mathrm{M}$ website views per year

At $\mathrm{BMC}$, research is always in progress.

Learn more biomedcentral.com/submissions 